\title{
Dynamic intelligent paging in mobile telecommunication network
}

\author{
S R PARIJA ${ }^{1, *}, \mathrm{~N} \mathrm{P} \mathrm{NATH}^{1}, \mathrm{P}_{\mathrm{K} \mathrm{SAHU}} \mathrm{S}^{1}$ and $\mathrm{S} \mathrm{S} \mathrm{SINGH}{ }^{2}$ \\ ${ }^{1}$ Department of Electrical Engineering, National Institutes of Technology Rourkela, Rourkela 769008, India \\ ${ }^{2}$ School of Electronics Engineering, KIIT University, Bhubaneswar 751024, India \\ e-mail: smita.parija@gmail.com; nilranjan.nath@gmail.com; pksahu@nitrkl.ac.in; sudh_69@yahoo.com
}

MS received 23 February 2015; revised 27 February 2017; accepted 4 August 2017; published online 10 March 2018

\begin{abstract}
The purpose of this work is to investigate the reduction in location management cost by profiling the subscriber with the sets of call data record (CDR) as inputs for a dynamic network simulation. We propose dynamic intelligent paging using the history of subscribers' behaviour directly from a CDR dataset along with knowledge of users' past location to predict their next location and thus reducing the paging resources. Simulated results with the actual user data show 4-5 times better performance than that of conventional paging and 3-4 times better than that of intelligent paging. The specific research contributions regarding the dynamic management algorithm, which can be used along with the existing system without any modifications, are outlined in this work. An illustrative scenario demonstrates the proposed approach with synthetic data. The novelty of this work is that instead of using theoretically predicted data it uses actual CDR data to profile the users.
\end{abstract}

Keywords. Mobility management; telecommunication network; intelligent profile-based paging; call data record (CDR); optimization of resources.

\section{Introduction}

In the current era, with advancement of mobile technology and consistent increase in mobile use the telecommunication network is gaining much more importance to maintain the quality of service (QoS). In the direction of enhancing QoS and narrowing the usage of bandwidth resources, an efficient dynamic location management technique is introduced. In the mobile personal communication services (PCS) during a call arrival to a mobile user, in order to maintain a good grade of service as well as for reliable communication it is required to track the current location of the mobile user, which results in frequent updation in the database [1].

In mobile telecommunication, the two major important embedment tasks, namely location function or location update and location search or paging of telecommunication network, need to be optimized to minimize the signalling cost. The location update procedure informs the network about the last known location of the mobile. In the paging procedure, the network tracks and queries about the user location to forward the call to a mobile user target location through BSC (Base Station Controller) and BTS (Base Trans-receiver) so that the mobile can receive the call. However, there is a trade-off between this location update and paging, which needs to be optimized as both consume

*For correspondence bandwidth. Location update and paging are inter-related as increase in frequency of location update will decrease the uncertainty in user's location; hence, paging needs to be done in a smaller area (and vice-versa). Hence, while focussing on optimization carefully, it should be considered so that the change in one should not affect the other in a negative manner.

However, this work focusses only on dynamic intelligent paging techniques, for which many literatures have been followed related to the location information, a significant attribute of location management.

It has been revealed that during the call arrival to a mobile target, call data record (CDR) traces and collects the location information of the subscribers' daily behaviour. This location information annotated from mobile phone triggers the researchers to work on various paging issues [2-4], which is the main focus of the work. This paging technique is a prime factor of location management where the network tracks human behaviour from the profile record and stored in Home Location Register (HLR) of mobile network.

In a wireless system, determining the exact location of the user, which is the main objective of location management, is done via two antagonizing techniques such as location update and paging [5]. However, for proper management of the network and solving management-related issues there exist two offline techniques, namely location areas (LAs) technique and Reporting Cell Planning 
Technique (RCPT). In the LA technique, when the user moves out to a new LA, there is updation in the location database, which keeps the user data that reflects the new base station. However, paging area (PA) consists of a cluster of cells receiving the paging messages through the paging procedure by the system to find the exact location of the user based on the last position of the cell where the user might be located.

The signalling overhead cost should be reduced through the paging process, which is not possible through the traditional paging scheme. In the traditional or conventional paging scheme all the cells are paged in a LA keeping in mind that the user may be located in a cell. As a result, signalling overhead increases by paging all the base stations in a LA [6-8]. Also, a larger number of cells in LA signifies the increase in signalling volume for paging while there would be decrease in the number of location updates and there exists a trade-off between location update and paging. Therefore, there are limitations of managing the signalling volume through the traditional location update and paging schemes. To overcome the issues of the traditional schemes, a lot of research works have been discussed in the literature to improve the system performances of location management scheme in the cellular network. However, many researchers have successfully worked on the personal user profile technique, which is a customized model of interest explicitly containing the user's personal behaviour.

Bhattacharya and Das [9] studied the human mobility pattern and in [10] the authors track the human behaviour based on intelligent paging strategy. Similarly, Akyildiz and Ho (1996) and other researchers [8, 11-13] proposed selective paging integrated with location update scheme with the assumption of call arrival procedure and cell residential time.

However, the present investigation is unique and organised in the following ways. Section 2 presents a related review of work based on profile-based paging. Section 3 gives an overview of mobile CDR data, study area and methodology used to analyse mobile phone records for the Bhubaneswar area. Also, how the CDR data are used for developing a new paging algorithm scheme based on users' profile replication for optimization of the resource utilization is discussed. In section 4, the proposed dynamic paging scheme is described in detail. Here, the performance of intersystem location management based on typical network parameters is analysed. Section 5 summarizes and validates the simulation results against independent database for the study area. Several numerical results of the location management schemes and comparison with the other existing paging techniques are also presented. Based on these findings, this work concludes with a discussion on the limitations and future work based on CDR database in the context of subscriber's behaviour.

\section{Related reviews}

Location management optimization is a prime issue in cellular network covering the geographical coverage LA that consists of many fixed base stations (BS) to offer high QoS. The terrain and radio coverage area is termed as a cell, where the fixed BS and number of subscribers are the principal components form a mobile cellular network.

The main task is proper management of the individual issue such as call mobility, mobility or location management and radio resource management in a cellular network. Among these issues, location tracking during a call arrival to the mobile user and collection of the position information is the main focus of the mobility management. In recent cellular management, location registration involves location tracking of the mobile user, which consists of a cluster of cells and is associated with two fundamental operations, which are location update and location search or paging counts, which generate the signalling load on the network. There is a trade-off between these two operations. However, this paper focusses only on the paging technique. With the insight of this knowledge, many studies have been investigated in the area of location management techniques; specifically, much attention has been focussed on paging procedure with the objective to reduce the signalling cost of mobile network. Work by [14] geared towards the new research direction or development of new location management schemes in support of enhancement of user density. To improve the performance of location management schemes, [15] proposed various alternative techniques. They also suggested a two-level hierarchical database structure, i.e., HLR and VLR (Visitor location register). HLR is a database of the cellular network that stores subscriber's profile permanently while VLR is a database that stores the user information temporarily and works during the roaming condition.

Roy et al [5] successfully developed various strategies for location inquiry and location update to improve performance of location management. Ref. [16] presents a cost-effective as well as signalling paging load in location management using several types of paging strategies. Wesolowski et al [17] deal with the profile-based paging based on users' previous location information to reduce the paging load. Similarly, Safa et al [18] also worked on signalling paging load; an optimum LA contains the fixed BSs and the subscribers. Moris et al [6] studied the user mobility pattern that provides the previous location information and extracts the behaviour of user for location management technique for static network. Wang et al [15] proposed an effective dynamic network management, profile-based LA and bandwidth factor.

Safa et al [18] also investigated on mobile phone data and location data table related to enhancement of the performance of the recent cellular network architecture. An extended search is proposed in [19] for the location 
Table 1. Call records of an individual ${ }^{\mathrm{a}}$.

\begin{tabular}{lcccrrr}
\hline Record type & Originating circle & Cell ID & Date & Time & Duration & Description \\
\hline MOC & 40472 & $04 f 467005 a 7942$ & 01-Aug-16 & $6: 48: 35$ AM & 28 & Voice call \\
MOC & 40472 & $04 f 467005 a 7941$ & 08-Aug-16 & $7: 37: 32$ AM & 35 & Voice call \\
MTC & 40472 & $04 f 467005 a 7941$ & 05-Aug-16 & $7: 38: 07$ AM & 62 & Voice call \\
MTC & 40472 & $04 f 467005 a 7941$ & 21-Aug-16 & $7: 58: 42$ AM & 24 & Voice call \\
SMT & 40472 & $04 f 467005 a 7941$ & 23-Aug-16 & $7: 56: 22$ AM & 1 & Message \\
SMT & 40472 & $04 f 467005 a 7941$ & 24-Aug-16 & $02: 10: 03$ PM & 1 & Message \\
SMO & 40472 & $04 f 467005 a 7938$ & 26-Aug-16 & $02: 15: 19$ PM & 1 & Message \\
SMO & 40472 & $04 f 467005 a 7 c b 1$ & 28-Aug-16 & $05: 10: 03$ PM & 1 & Message \\
\hline
\end{tabular}

${ }^{\mathrm{a}}$ The columns from left to right represent the record type ( $M O C$ mobile-originated call, $M T C$ mobile-terminated call, $S M T$ subscriber message terminated, SMO subscriber message originated), originating circle, i.e., location area, cell ID, which represents the user cell id, the location of the BS, the date, time and duration (in minutes) of the call activity. Here call activity includes voice call and message

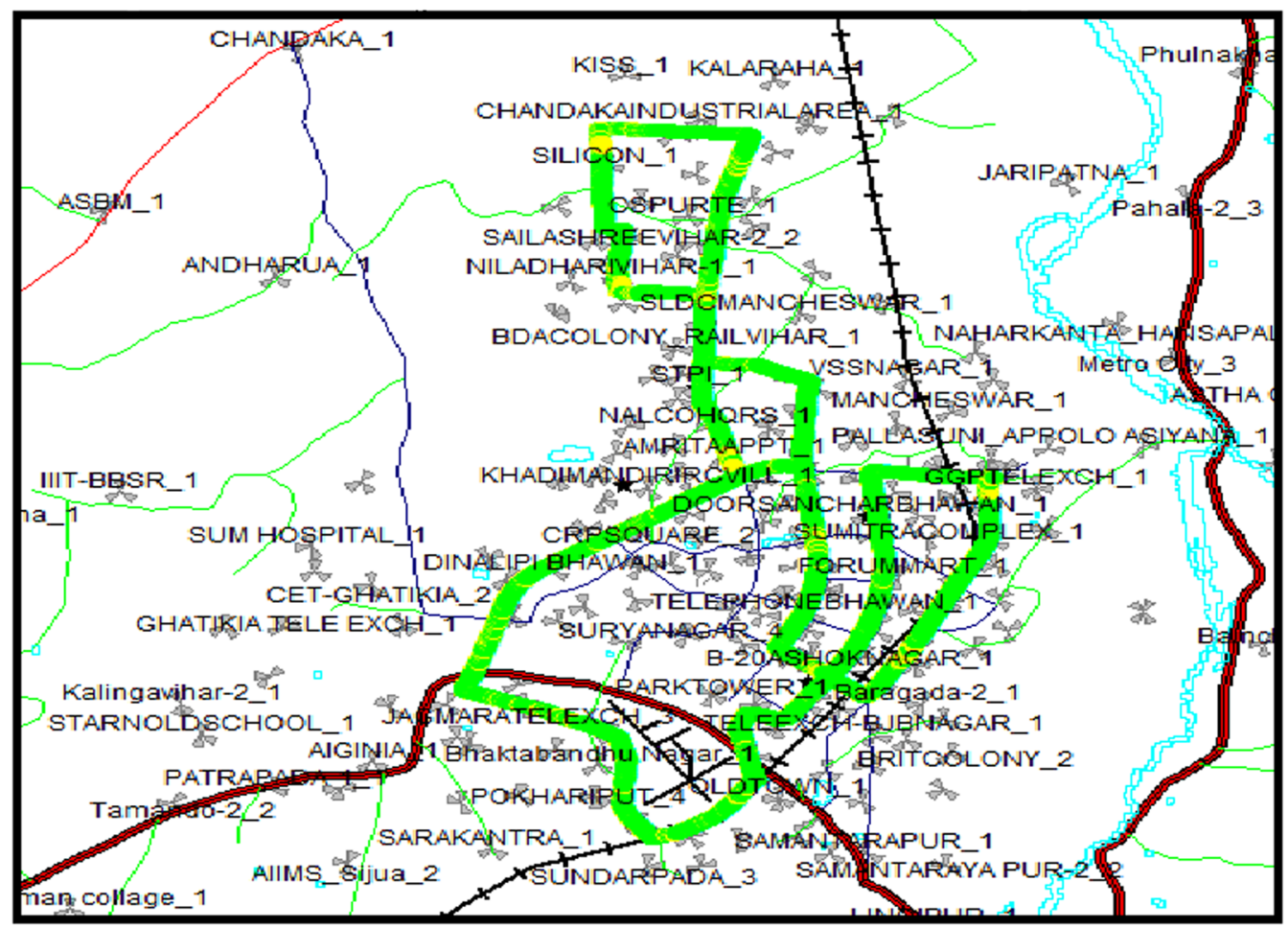

Figure 1. Visualization of base station coverage in study area BBSR.

procedure where the called mobile is paged first, which resides in the mobile network prior to the search in whole LA. A scheme to extract the trajectory pattern of mobile user in order to guarantee QoS is suggested in [1, 19]. Various theoretical approaches are proposed based on profile-based paging investigated by the researchers [20-22] and the parameters such as degree of mobility, user's motion process, call arrival process, etc. are explored. However, a novel profile-based paging classical method is proposed to reduce the signalling cost also, there is a work on memory method by the introduction of an intelligent paging procedure. Wang et al [23] depicted another intelligent paging procedure known as Hypo-LA (Hyper-Location area) paging in GSM-EDGE Radio Access Network (GERAN), where the Hypo-LA paging is segmented into small groups called PAs. This paging group also takes the previous location information of mobile user from the known last cell location. Using this information, which helps in the paging process, becomes easier and reduces paging load. When the paging process fails, sequential paging is performed with the help of other cells (or PAs). Wang et al $[15,23]$ worked on sequential and blanket paging and also compared the two paging algorithms. 
Zang and Bolot [24] and Misra et al [25] investigated on CDR data generated by a mobile phone to improve paging performance with a better location management scheme. This work calculates the probability of weightage of users based on their movement and correlation with their previous movement. With this concept, the present work focusses on the periodicity and an approach driven by personal mobility to simulate the proposed issue. Finally, the work proposed in [26-30] focused on dynamic management that is feasible using different technologies such as mobile pone tracing, intelligent paging strategy, profile based paging and the smartphone based human mobility prediction. Here, profiles are created by exploring and using the periodicity in the users' movement. It is observed that more than $90 \%$ of the mobile users visit only $1 / 3$ rd of the cells present in a location. Also, according to time and place, most of the cellular users show periodicity in their movement pattern. That is, many of the consumer's everyday activities are carried out in a routine manner in terms of time and space. Hence, whether it is an employee or a student, he continues to go to the office (or university) and returns home at the same (i.e., with slight to no variation) time. However, in the case of highly mobile users, such as a salesman (or taxi driver) the path taken is different and the group of cells visited most usually is not consistent.

\section{Mobile CDR data, study area and methodology}

Performance evaluation is assessed through simulation using collected real CDR database for exploring the proposed algorithm. The CDR data annotated from mobile phone contain the daily behavioural pattern available at
MSC (Mobile Switching Centre), BSNL, BBSR, Odisha. This CDR database, used for billing purpose, is shown in table 1. This work is simulated on the central part of BBSR area using the database of 800 mobile users in a 4-month duration. Figure 1 visualizes the BBSR area considered for implementation of the proposed algorithm. The marked geographic coverage zone shows a particular node considered for study area.

\subsection{Mobile CDR database}

The CDR database used for designing the algorithm is in the form of user, day and time shown in figure 2. User days are considered as Monday, Tuesday, etc. Further, weekends days are included, for which they are segmented into slots of $1 \mathrm{~h}$ as per the time of the day in a 24-h structure. Each slot contains three parameters.

(1) location (loc): It is in the form an array that stores the cell that the user visited for the period of that hour.

(2) Weight ( $w t)$ : It is also an array of equal length as that of location array. It stores the weights of the cells based on their correlation with previous measurements. Each cell location is assigned a probability of weightage correlated to the previous measurements. With each update, the weightage value increases or decreases depending on user mobility in that LA. For example, a user with higher weightage value indicates the availability of user in that cellular area, or else the weight value decreases [26].

(3) History $(h t)$ : It signifies the number of times the user is visited in the identical LA. Based on the history of the

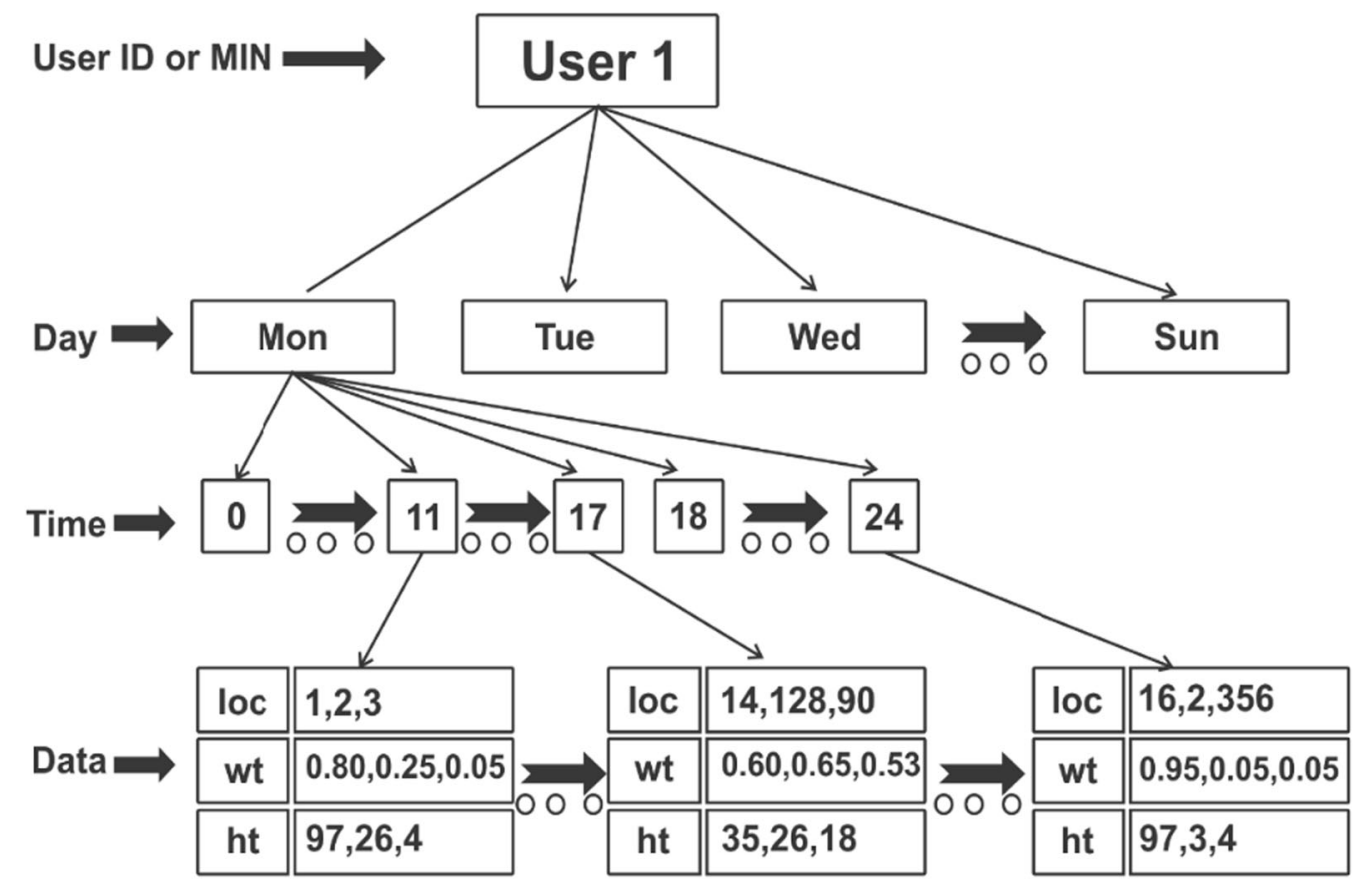

Figure 2. CDR database of mobile user. 


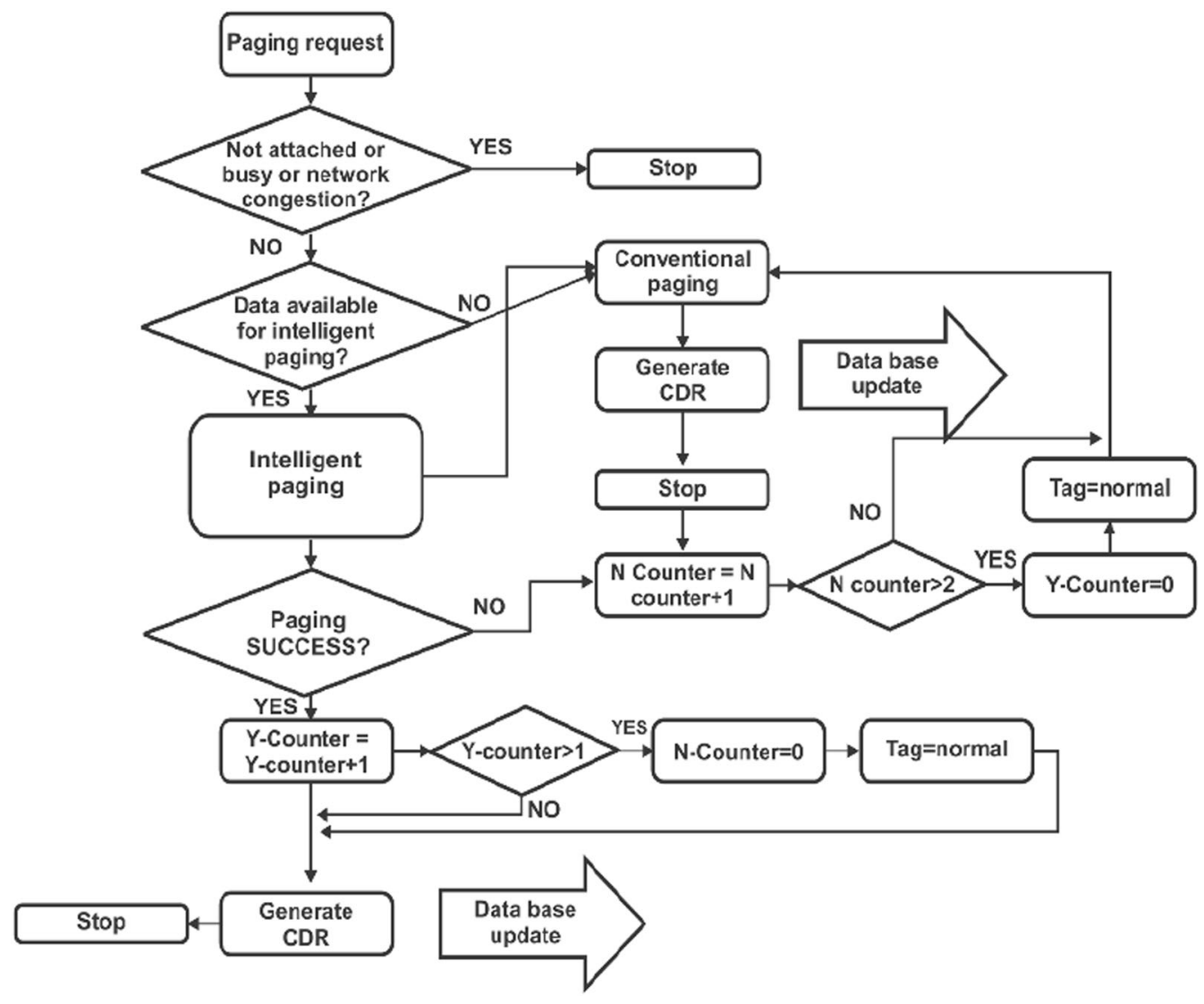

Figure 3. Flow chart of paging algorithm.

user's movement, MSC easily investigates the cell location and accordingly pages that area. This information is placed in a structure. Hence, individual user can be identified by its structure with its Mobile Identification Number (MIN). The generalized structure is shown in figure 2 .

\subsection{Correlating data and updating}

The mobile user CDR data are correlated with the users' previous movement and accordingly weight vector is calculated with each and every update. The correlation is obtained for the particular day and time. The update data are stored in a structure similar to that of the data. With the same data, simulations are carried out to check whether the cell is (or cells are) present in the update data in the structure of day time slot or not $[27,28]$.

If the cell is present:

If the cell is present then $h t$ corresponding to the loc is incremented and then new weight $w t$ is calculated. Let $l o c$ ( $n$ ) be the cell that is found to be present. Then wt corresponding to $l o c(n)$ shall be calculated as $w t(n)=\frac{[(\text { user.day.time.wt }(n)) *(\text { user.day.time } . h t(n))]+t w}{\left[\sum(\text { user.day.time. } h t)\right]+t h}$

$$
h t(n)=h t(n)+t h
$$

and for other $l o c$, i.e., $\operatorname{loc}(m)$, where $m \neq n$

$$
w t(m)=\frac{(\text { user.day.time.wt }(m)) *(\text { user } . \text { day.time } . h t(m))}{\left[\sum(\text { user.day.time } \cdot h t)\right]+t h}
$$

$$
h t(m)=h t(m)
$$

where $t w$ stands for temporary weight and is computed by dividing 1 by the total number of distinct cells visited by the user in that hour. In this work th stands for temporary history calculated similar to $t w$; they both have the same numerical value. Hence, during the time when the user is not moving at all or is moving slowly, $t w$ and $t h$ values will be $\approx 1.0$ and hence $w t$ of $l o c(n)$ is increased while weight of other local is decreased gradually. However, during the time when user movement is excessive, like travelling, etc. or in case of a highly mobile user, $t w$ and $t h$ values will be small. Hence, although $w t$ of $\operatorname{loc}(n)$ will be higher than the 
others, still the total value of $w t$ will decrease. Hence, the sum of the $w t$ array will also decrease, which will help in determining the cells to be paged and is described in further discussion.

If the cell is not present:

In this case the new cell is added to loc array; its $w t$ and $h t$ are also added to the respective arrays calculated as follows:

$$
\begin{gathered}
w t(\text { new })=\frac{t w}{\left[\sum(\text { user.day.time } . h t)\right]+t h} \\
h t(\text { new })=t h
\end{gathered}
$$

For the rest of the loc, i.e., $\operatorname{loc}\left(m^{\prime}\right)$ where $m^{\prime} \neq$ new

$w t\left(m^{\prime}\right)=\frac{\left[\left(\text { user.day.time.wt. }\left(m^{\prime}\right)\right) *\left(\text { user.day.time. } h t .\left(m^{\prime}\right)\right)\right]}{\left[\sum(\text { user.day.time.ht })\right]+t h}$

$$
h t\left(m^{\prime}\right)=h t\left(m^{\prime}\right)
$$

For a small weight value of the new cell, if loc(new) is just a temporary de-route from the regular cycle then its weight will be reduced and soon it will be removed from the loc list. However, if this is not the case then the $w t$ value will be increased with successive input and it will be regarded as an important cell to be paged [29, 30]. It can be inferred that if the value of $(w t \times h t)$ decreases below a certain value (0.0001) then the loc along with all the elements is deleted.

\section{Location search or proposed paging method}

Location search or paging method is an important entity of mobility management that is used to locate the mobile terminal, which is performed by the network during the location inquiry. Normally, this paging technique is performed at the MSC based on the users' last known location. This search procedure is directly related to the user profile database. The paging technique is directly related to the number of incoming calls to a cell. MIN is a key term that identifies the user, on which MSC performs the paging operation. Basically, profile-based paging operation is carried out by MSC; if the network fails to get MIN then conventional paging, i.e., blanket paging is performed.

The proposed paging flow chart is shown in figures 3,4 , 5. If the number of times the user visits a LA is large, then $w t$ value of that LA increases. Accordingly it shows better paging performance and sum total of the $w t$ of the location area paged provides more than $95 \%$. The reason behind this algorithm confirms that paging delay is the main component of achieving better paging success rate. As per the proposed method, if in case the first page fails then the chances of finding the user in all the cells are more than those during second paging. In addition, if still paging is not

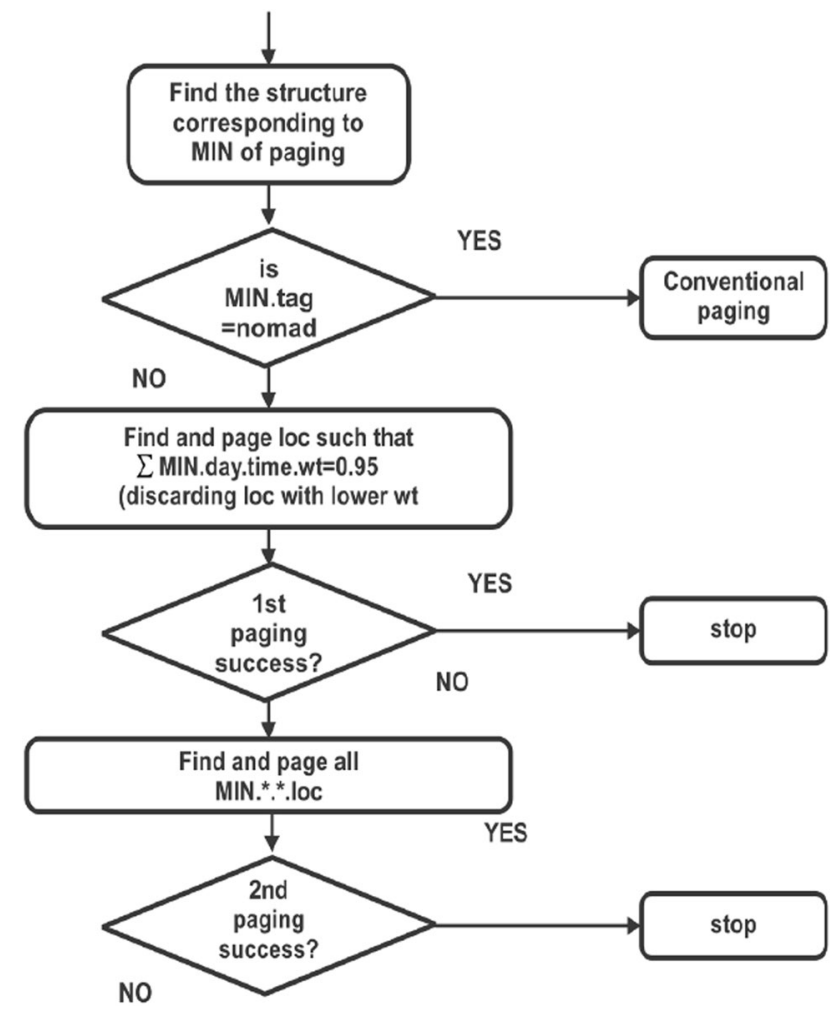

Figure 4. Intelligent paging flow chart.

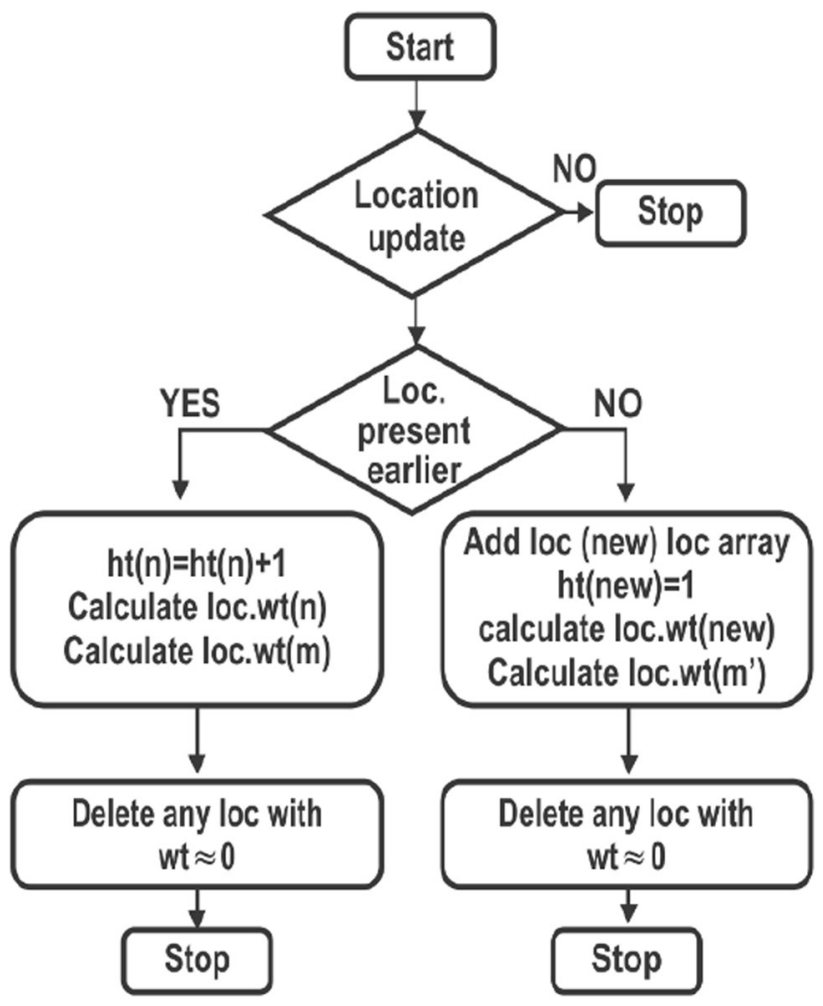

Figure 5. User data structure update flow chart. 


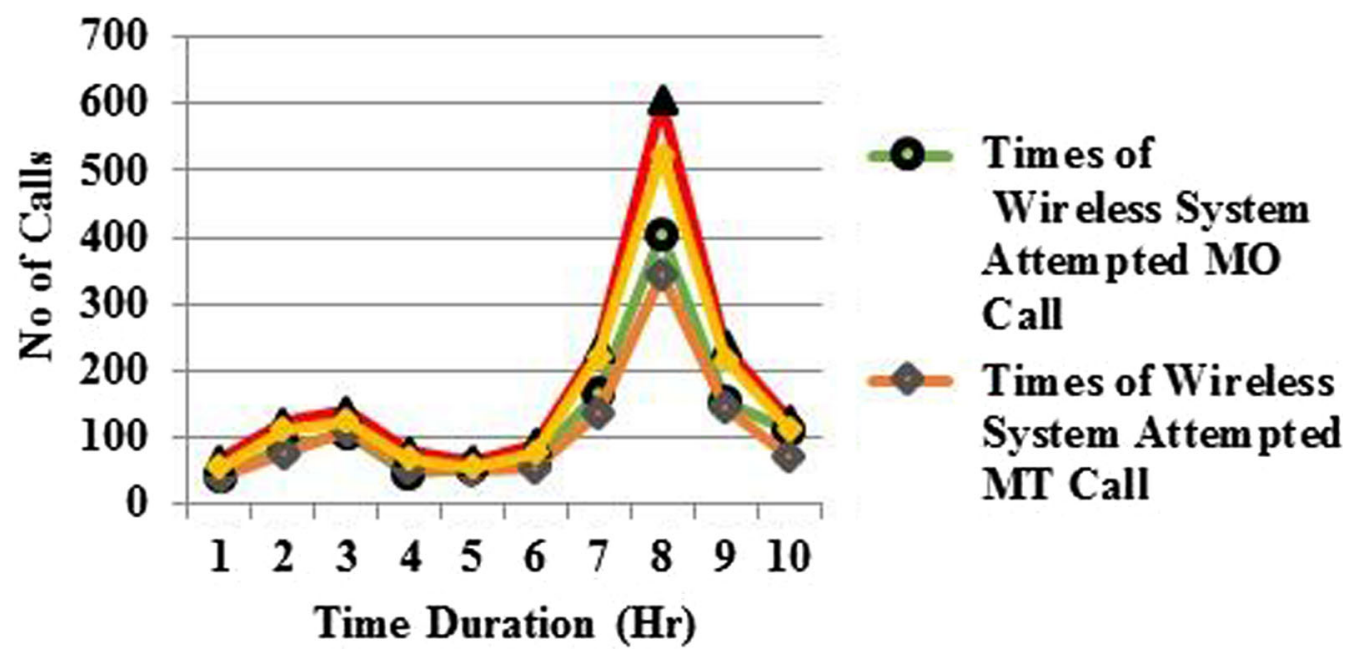

Figure 6. Frequency of calls on a day.

successful then blanket paging is carried out in that LA, which is a conventional paging.

\section{Simulation results}

This section presents performance evaluation of profilebased paging as well as a detailed analysis of the present analytical model to assess the efficiency and accuracy. The proposed profile-based paging algorithm captures all relevant aspects of the proposed approach in a concise way. The simulation model considers both call delivery, call arrival and mobility behaviour to offer various services of mobile cellular network. In this work three types of users such as highly mobile, moderately mobile, less mobile and average users are considered. The simulation experiment was conducted over 3 months from 1st August 2016 to 1st October 2016 within the Bhubaneswar area comprising 1000 mobile towers. For the sake of brevity, the call arrival and call delivery scenario in a particular day (1st August 2016, randomly selected) is plotted. The frequency of calls against the time duration is shown in figure 6 , which presents the number of calls attempted by the system to the number of calls terminated. The 4 months CDR data are explored in MATLAB 6.5, out of which 1 month data are used for training the system and other 2 months data are used for validation purpose. The outcomes of the proposed algorithm are taken into consideration and shown in figures $7,8,9$.

\subsection{Comparison analysis of various users}

This section furnishes the detailed paging characteristics of different mobile users as test cases using the CDR data. To further analyse the evaluations, the performance analysis of the proposed method for different users is thoroughly presented. The performance of paging process is interpreted in terms of percentage of paging success rate over 3 months duration. In addition, the possible ways to express the outcomes for four types of users such as less mobile user, moderately mobile user, highly mobile user and averagemobile user are depicted in the work. Some results with accurate extraction of information from the evaluation survey are presented, which was conducted for various respondents.

5.1a Less mobile users: Less mobile users are the most sedentary users confined to one LA. Consequently, the number of cells visited by such type of users is less as they are less mobile. Less mobile users include professional persons like workplace employees, students, doctors, house wives, etc. They are about $45-60 \%$ of the total population, whose movements are ordinarily characterized by limited and periodic action. Accordingly, they show the highest paging success rate. In the first week the paging performance is $50 \%$ in the first paging, which then steadily increases to over $93 \%$ at the end of the 12th week in the second paging. Even though the system is properly trained, during second paging, there is not much improvement, which is depicted in figure 8 . The paging performance observed is from $50 \%$ to $93 \%$ success rate. From table 2 it is inferred that the number of cells paged is 25 and during second paging the number of cells attempted to page is $37-45$.

5.1b Moderately mobile users: The movement rate of moderately mobile users is basically more than that of less mobile users and less than that of highly mobile users. Hence, their randomness is moderate; $35-45 \%$ of total population is moderately mobile users, which constitutes the people like office workers, foot merchants (whose work place is at a distance from the residence) 


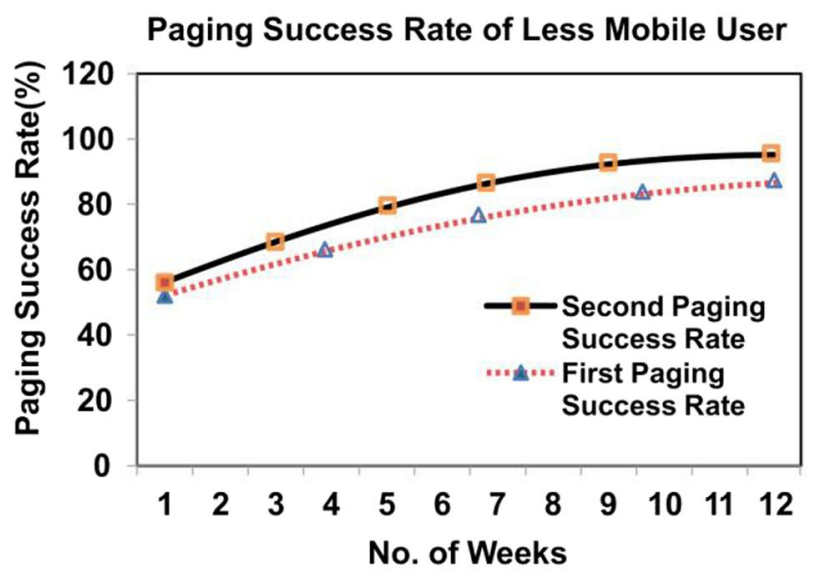

Paging Success Rate of Highly Mobile User

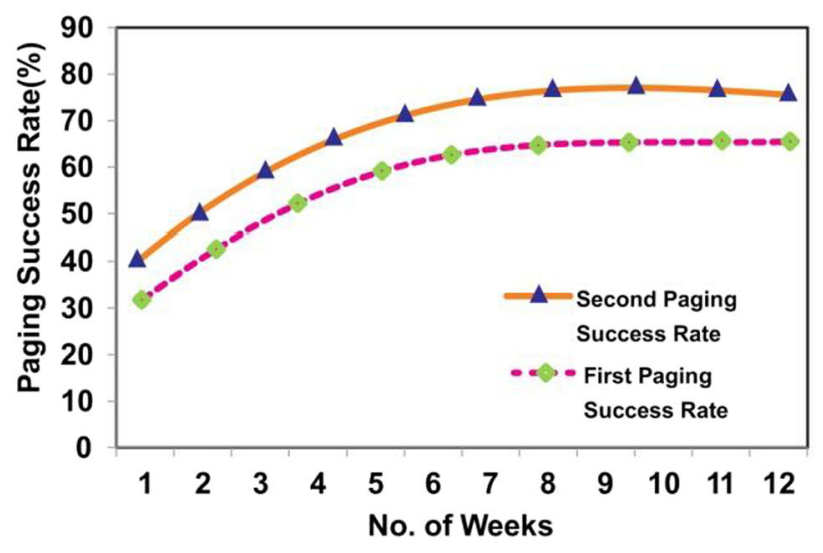

Paging Success Rate of Different Users (First Paging)

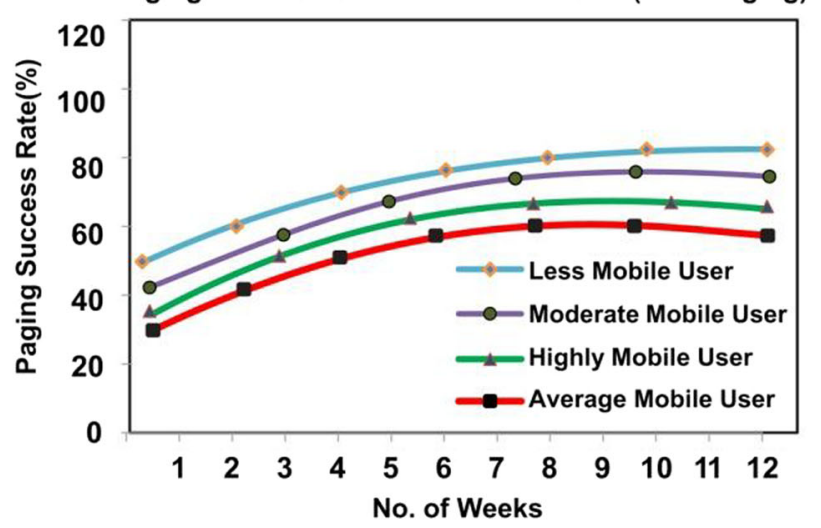

Figure 7. Paging success rate after first paging.

and so on. Generally, there movement frequency is about a couple of areas. For such type of moderate users the result analysis shows that the paging success rate starts at $43 \%$ and ends at $78 \%$ with an improvement rate less than that of low-mobile users and more than that of the highly mobile users. It is revealed from figure 8 that the outcome of second paging is from $45 \%$ to $82 \%$. From table 2 it is observed that the number cells paged by the
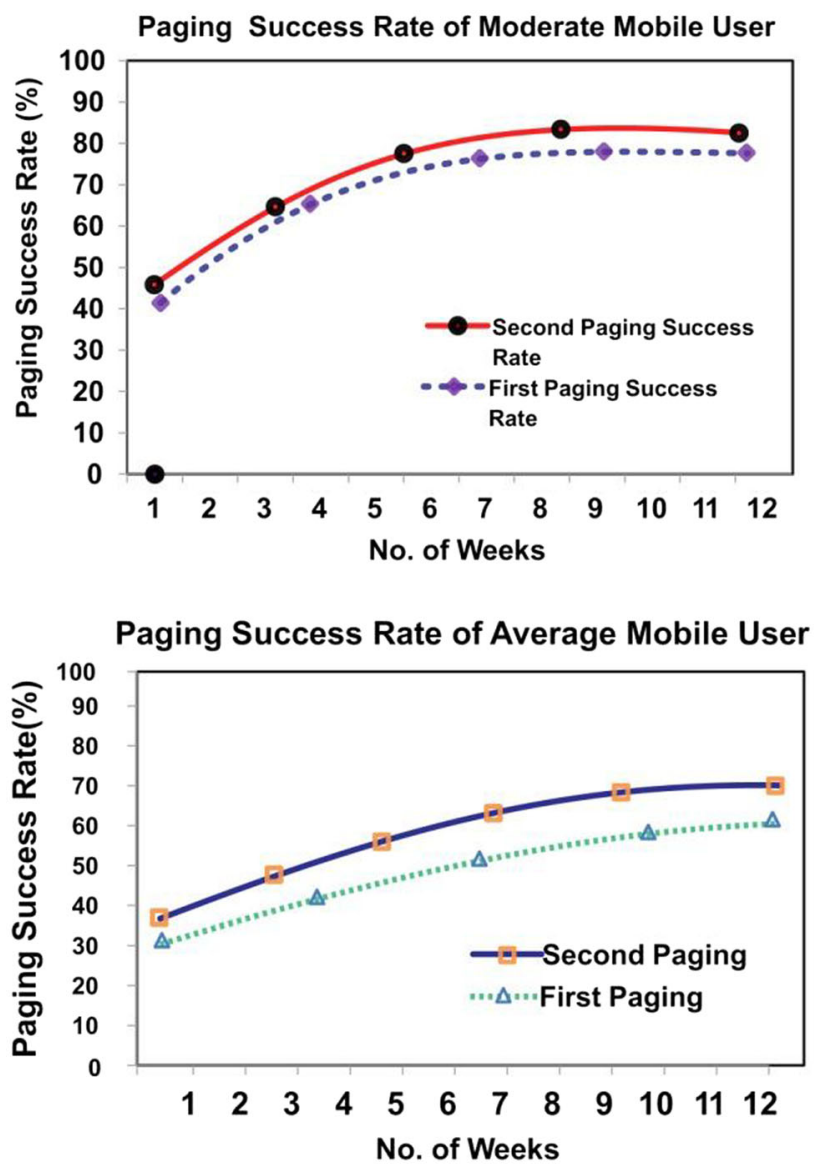

Paging Performance of different users(Second Paging)

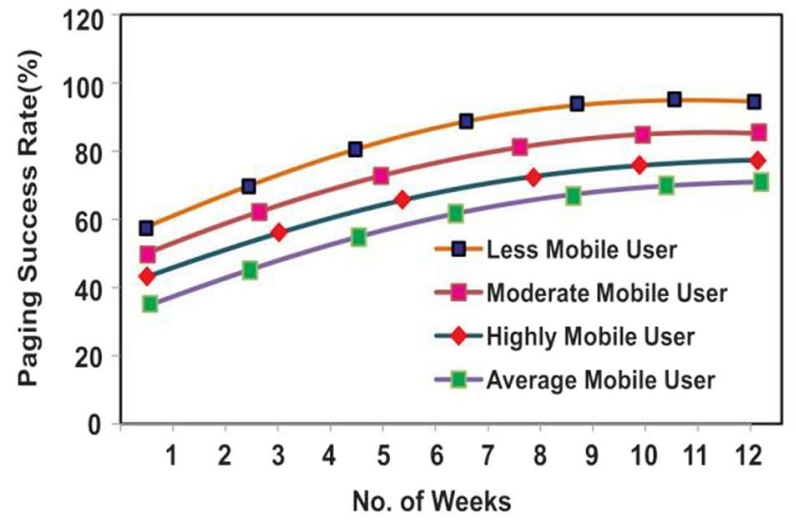

Figure 8. Paging success rate after second paging.

network is 44 and 83 during the first and second paging, respectively.

5.1c Highly mobile users: They are the users who travel more than three LAs and comprise up to $10-30 \%$ of the total population. The users like cab drivers, sales representatives, merchants and so on come under highly mobile users category. Their movement patterns are often random and not periodic. Hence, the paging success rate starts at 


\section{Comparative Study of Paging Success Rate of Different Types of Users}

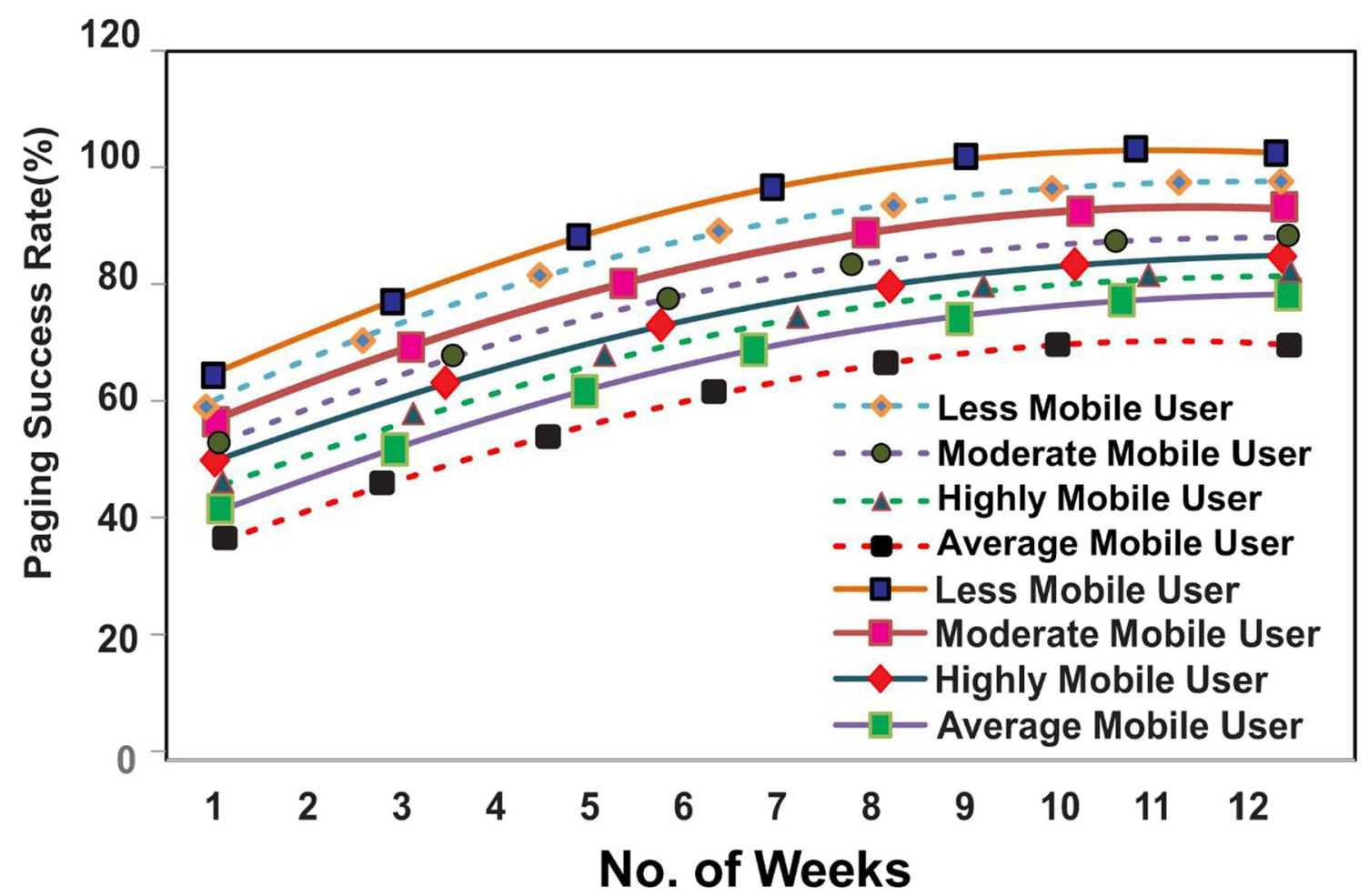

Figure 9. Paging success rate after first paging and second paging of all types of users and their average.

mere $32 \%$ and settles at just above $65 \%$. However, from table 2 it is seen that in the second paging, the success rate rapidly increases from $40 \%$ to $75 \%$ towards the end of 12 weeks. It is also observed that the maximum number of cells paged for a paging request during first paging is 95 and that in the second paging the cell number is 174 (tables 2 and 3).

\subsection{Paging success rate of different users}

It is observed from table 2 that on an average, paging success rate increases nearly $41 \%$ in the first week during first paging while in second paging it increases to $85 \%$ over the 12 th week and then it reaches $93 \%$ for second paging. It is revealed from the analysis that such type of paging technique saves a lot of bandwidth when compared with other intelligent paging methods. Table 4 shows a comparison analysis of different paging strategies such as Hypo-LA paging, proposed paging and conventional paging to present the number of cells paged per paging request.

It can be clearly observed from table 3 that paging success rate is $95 \%$ for conventional paging, better when compared with Hypo-LA paging, and the paging success rate is $83.94 \%$. However, for the proposed paging algorithm the paging success rate is $85 \%$, whose performance is not as good as that of conventional paging but better than that of the Hypo-LA paging. As a result it clearly highlights the superiority of the proposed algorithm, which not only saves the bandwidth but also reduces the paging load by paging less number of cells and hence increases the paging success rate. Similarly, it is seen from table 4 that the number of cells paged for moderately mobile users is 44 cells in the first paging for proposed paging and the performance furnishes a better result, around 47 cells paged, while in case of Hypo-LA paging the number of cells paged is about 200-400 cells. In case of highly mobile users for proposed paging the number of cells paged is 95 , which increases to 174 during second paging. For the same highly mobile users, the number of cells paged for Hypo-LA paging is 200-400 cells.

It can be summarized from table 3 that as there is not much improvement in second paging for the less mobile, in case of excessive paging load on MSC, second paging can be skipped and paging delay can be improved. In this way, improvement in paging delay and paging success rate can be achieved. As the less mobile users are large in number, this improvement of paging delay will help minimize the congestion in MSC, which is helpful during high paging load; as a result, it decreases the holding time for other users.

Similarly, it is also observed that the outcomes of first paging for highly mobile users are not good; as second 
Table 2. Success rate data for different types of users in different weeks.

\begin{tabular}{|c|c|c|c|c|c|c|c|c|}
\hline \multirow[b]{2}{*}{$\begin{array}{l}\text { Week } \\
\text { no. }\end{array}$} & \multicolumn{4}{|c|}{ First paging } & \multicolumn{4}{|c|}{ Second paging } \\
\hline & $\begin{array}{c}\text { Less mobile } \\
\text { user }\end{array}$ & $\begin{array}{l}\text { Moderately } \\
\text { mobile user }\end{array}$ & $\begin{array}{l}\text { Highly mobile } \\
\text { user }\end{array}$ & Average & $\begin{array}{c}\text { Less mobile } \\
\text { user }\end{array}$ & $\begin{array}{l}\text { Moderately } \\
\text { mobile user }\end{array}$ & $\begin{array}{l}\text { Highly mobile } \\
\text { user }\end{array}$ & Average \\
\hline Week 1 & 48 & 45 & 32 & 41 & 50 & 48 & 32 & 43 \\
\hline Week 2 & 52 & 49 & 35 & 45 & 55 & 53 & 42 & 50 \\
\hline Week 3 & 59 & 49 & 37 & 49 & 63 & 53 & 40 & 52 \\
\hline Week 4 & 66 & 51 & 44 & 54 & 70 & 55 & 52 & 59 \\
\hline Week 5 & 73 & 61 & 59 & 65 & 77 & 65 & 65 & 69 \\
\hline Week 6 & 78 & 68 & 64 & 70 & 82 & 72 & 70 & 75 \\
\hline Week 7 & 86 & 73 & 66 & 75 & 90 & 77 & 72 & 81 \\
\hline Week 8 & 88 & 75 & 68 & 77 & 92 & 83 & 79 & 85 \\
\hline Week 9 & 91 & 77 & 67 & 79 & 95 & 80 & 77 & 84 \\
\hline Week 10 & 93 & 83 & 65 & 81 & 97 & 89 & 75 & 87 \\
\hline Week 11 & 96 & 84 & 66 & 82 & 98 & 90 & 80 & 91 \\
\hline Week 12 & 91 & 82 & 72 & 85 & 94 & 88 & 86 & 93 \\
\hline
\end{tabular}

TMSI Temporary Mobile Subscriber Identity, IMSI International Mobile Subscriber Identity, LA location area, BSC Base Station Controller.

Table 3. Comparison among conventional paging, Hypo-LA paging and proposed paging.

\begin{tabular}{lccc}
\hline & $\begin{array}{c}\text { Normal } \\
\text { paging }\end{array}$ & Hypo-LA paging & $\begin{array}{c}\text { Proposed } \\
\text { paging }\end{array}$ \\
\hline $\begin{array}{l}\text { First paging } \\
\text { type }\end{array}$ & TMSI & TMSI & TMSI \\
$\begin{array}{l}\text { First paging } \\
\text { area }\end{array}$ & LA (600 & BSC (315 cells on & 38 cells \\
$\begin{array}{l}\text { Success rate } \\
(\%)\end{array}$ & 95 & $\begin{array}{c}\text { Avg. }) \\
83.94\end{array}$ & 85 \\
$\begin{array}{l}\text { Second paging } \\
\text { type }\end{array}$ & IMSI & IMSI & IMSI \\
$\begin{array}{l}\text { Second paging } \\
\text { area }\end{array}$ & LA (600 & LA (600 cells) & 136 cells \\
$\begin{array}{l}\text { Success rate } \\
(\%)\end{array}$ & 88.62 & 89 & 93 \\
\hline
\end{tabular}

paging includes all the cells, first paging can be skipped, paging load can be reduced and paging delay can be improved. Similarly, the bandwidth conservation is less for highly mobile users as the number of cells paged for the highly mobile users in the second paging is very high.

A provision could also be added that even with the less available data, in order to improve the paging success rate, all LAs, which consist of 3 BTS, can be paged during the initial weeks. After the simulation, overall average success rate saturating around $89.33 \%$ (approximately) can be achieved for a typical paging scenario.

5.2a Prediction results for different users: To assess the performance evaluation, the statistical measures such as mean, median, mode, confidence interval (95\%), etc. have been carefully considered in this paper. Prediction accuracy
Table 4. Comparison of proposed paging and Hypo LA paging on the basis of number of cells paged.

\begin{tabular}{cccc}
\hline & \multicolumn{2}{c}{ Proposed paging } & \\
\cline { 2 - 3 } Type of user & $\begin{array}{c}\text { First } \\
\text { paging }\end{array}$ & $\begin{array}{c}\text { Second } \\
\text { paging }\end{array}$ & Hypo-LA paging \\
\hline Less mobile & 25 cells & $37-45$ cells & 1 BSC (200-400 cells \\
& (max.) & (max.) & approx.) \\
Moderately & 44 cells & 83 cells & 1 BSC (200-400 cells \\
mobile & $($ max.) & (max.) & approx.) \\
Highly & 95 cells & 174 cells & 1 BSC (200-400 cells \\
mobile & $($ max.) & (max.) & approx.) \\
\hline
\end{tabular}

in terms of paging success rate based on standard deviation is calculated for Less mobile user, Highly mobile user, Moderately mobile user and Average mobile user is shown in figure 10. It is illustrated from table 5 that as the system is not properly trained, for less mobile users, as they are less mobile, in the first paging, less number of cells are paged than in second paging. The prediction accuracy is measured in terms of standard deviation, which is more in first paging than in second paging. In second paging, as the system is properly trained, standard deviation is less in case of less mobile users when compared with moderately and highly mobile users. The reason behind the low standard deviation of less mobile users is related to their lesser randomness of the mobile users compared with moderately and highly mobile users.

Moderately mobile users move more number of cells than less mobile users and the cell number is less compared with highly mobile users. It is seen from table 5 that during second paging the system is properly trained and hence 

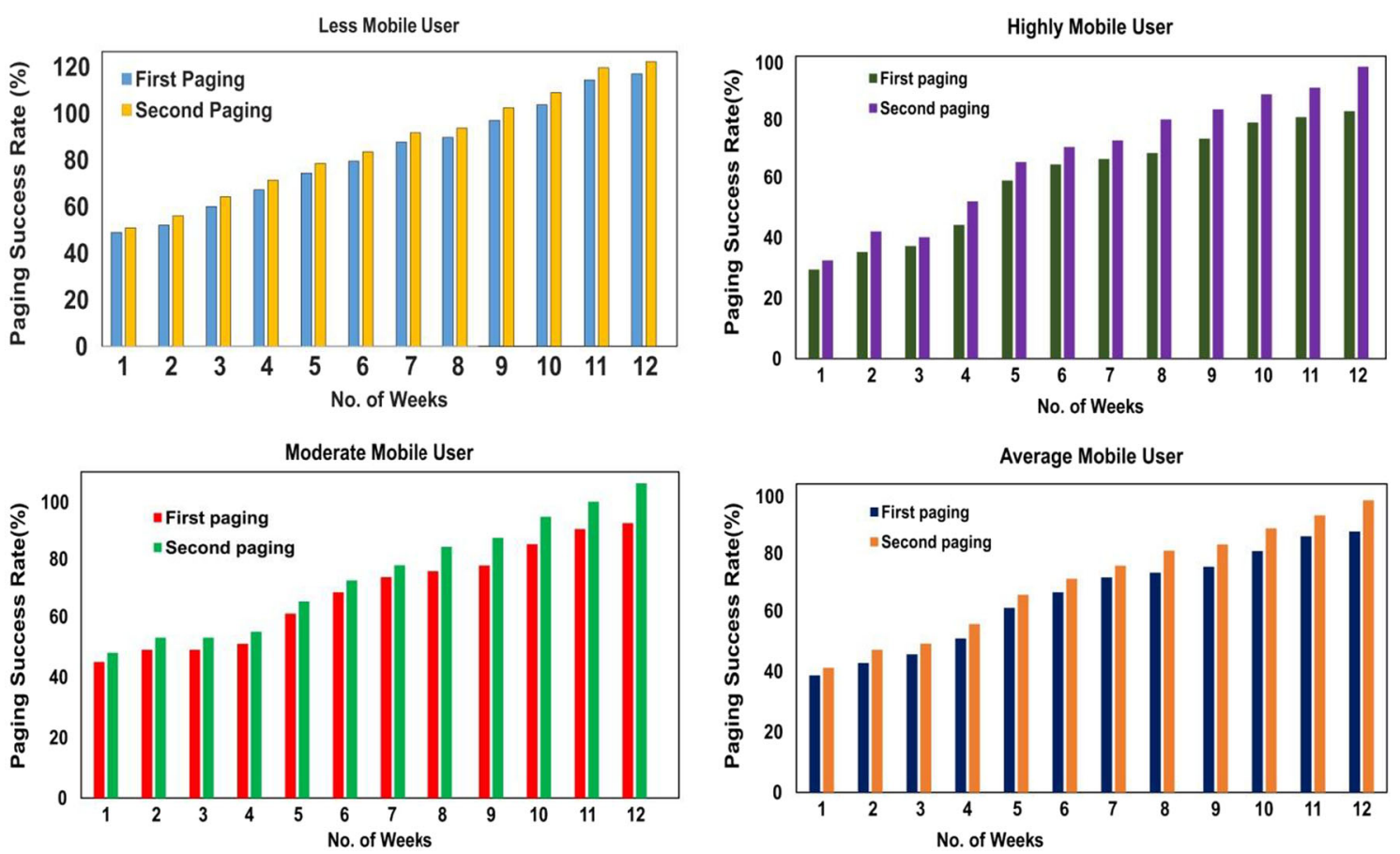

Figure 10. Accuracy measurement of different types of users.

Table 5. Accuracy measurement of different users.

\begin{tabular}{|c|c|c|c|c|c|c|}
\hline & \multicolumn{2}{|c|}{ Less mobile user } & \multicolumn{2}{|c|}{ Moderately mobile user } & \multicolumn{2}{|c|}{ Highly mobile user } \\
\hline & First paging & Second paging & First paging & Second paging & First paging & Second paging \\
\hline Mean & 82 & 84 & 69 & 75 & 60 & 69 \\
\hline Standard error & 4 & 4 & 3 & 4 & 4 & 5 \\
\hline Median & 90 & 93 & 76 & 81.5 & 66 & 76 \\
\hline Mode & 91 & 97 & 49 & 53 & 66 & 83 \\
\hline Standard deviation & 16 & 17 & 140 & 15 & 15 & 17 \\
\hline Sample variance & 27 & 270 & 197 & 240 & 233 & 312 \\
\hline Range & 49 & 49 & 39 & 45 & 46 & 54 \\
\hline Minimum & 48 & 50 & 45 & 48 & 29 & 32 \\
\hline Maximum & 97 & 99 & 84 & 93 & 75 & 86 \\
\hline Sum & 1298 & 1351 & 1117 & 1205 & 967 & 1103 \\
\hline Count & 16 & 16 & 16 & 16 & 16 & 16 \\
\hline Confidence level (95.0\%) & 9 & 9 & 7 & 9 & 8 & 9 \\
\hline Lower & 72 & 75 & 62 & 67 & 52 & 80 \\
\hline Avg. & 84 & 89 & 70 & 76 & 60 & 78 \\
\hline Upper & 90 & 93 & 77 & 84 & 68 & 93 \\
\hline
\end{tabular}

standard deviation on an average decreases for every iteration. Similarly, for highly mobile users, the randomness is more, and the users cover more number of cells in a LA. It is observed that the resources get optimized in second paging and the outcome is better for less mobile users than moderately mobile and highly mobile users. The database is designed in such a way that the algorithm adapts to the dynamic nature of mobile user movement and as a result provides better performance towards the end of last week of fourth month.

\section{Conclusion and future work}

In this paper, efficient utilization of profile-based technique for wireless mobile environment in relation to individual's daily behavioural pattern imposed by collected CDR data is proposed. The proposed strategy involves the spatial behaviour of individual user over a period of 3 months. The large-scale collected CDR data are based on several measures such as week, day and time. The prime objective is to develop the system where the subscriber is paged using 
profile-based paging based on the last updated location. The system sequentially pages each location of the users of the cell in a network. The CDR data usage through mobile phone annotation is fairly simple, economic and involves low collection cost. Besides these, the result obtained from this work confirms the efficiency and effectiveness of profile-based method and it is better for all dynamic cellular networks.

This paper deals with the proposed paging algorithm, which explores a new attribute to exploit a better paging characteristic. This work has taken a data-driven approach to optimize paging characteristics. The algorithm has been designed and tested in such a way that any network operator who wants to implement can do so with a stability period of 5-6 weeks. The algorithm is self-sufficient and it adapts to change in the user's movement pattern. In this way it makes the proposed algorithm a truly intelligent paging algorithm. The proposed algorithm increases the efficiency of bandwidth utilization with little to no effect in overall paging success rate.

The present work can be extended for further improvement in the proposed paging scheme. It can be explored for other than cellular networks such as UMTS, LTE network. Also, CDR data is used not only for implementation of paging technique but also integrate the location update and optimize the same issue with the hybrid optimization algorithm as a future work for dynamic network management.

\section{References}

[1] Abbass H A and Green D G 2012 Motif difficulty (MD): a predictive measure of problem difficulty for evolutionary algorithms. Evol. Comput. 20(3): 321-347

[2] Schneider C M, Belik V, Couronné T, Smoreda Z and González M C 2013 Unravelling daily human mobility motifs. J. R. Soc. Interface 10: 20130246, https://doi.org/10. 1098/rsif.2013.0246

[3] Gonzalez M C, Hidalgo C A and Barabási A L 2008 Understanding individual human mobility patterns. Nature 453: 779-782, https://doi.org/10.1038/nature06958

[4] Wang H, Calabrese F, Di Lorenzo G and Ratti C 2010 Transportation mode inference from anonymized and aggregated mobile phone call detail records. In: IEEE Conference on Intelligent Transportation Systems, Proceedings, ITSC, pp. 318-323, https://doi.org/10.1109/itsc.2010.5625188

[5] Roy A, Misra A and Das S K 2007 Location update versus paging trade-off in cellular networks: an approach based on vector quantization. 6(12): 1426-1440

[6] Morris D and Aghvami A H 2006 A novel location management scheme for cellular overlay networks. IEEE Trans. Broadcast. 52(1): 108-115

[7] Wang W, Akyildiz I F and Stuber G L 2000 An optimal partition algorithm for minimization of paging costs. Globecom 'OO - IEEE. Global Telecommunications Conference, Conference Record Cat. No. OOCH37137, vol. 1, pp. 188-192, https://doi.org/10.1109/glocom.2000.891748
[8] Pollini G P 1997 A profile-based location strategy and its performance. IEEE J. Select. Areas Commun. 15(8): $1415-1424$

[9] Bhattacharya A and Das S K 1999 LeZi-update: an information-theoretic approach to track mobile users in PCS networks. https://doi.org/10.1145/313451.313457

[10] Lyberopoulos G L, Markoulidakis J G, Polymeros D V, Tsirkas D F and Sykas E D1995 Intelligent paging strategies for third generation mobile telecommunication systems. IEEE Trans. Veh. Technol. 44: 543-554, https://doi.org/10. 1109/25.406621

[11] Akyildiz I F and Ho J S M 1996 Movement-based location update and selective paging for PCS networks. IEEE/ACM Trans. Netw. 4(4): 629-638, https://doi.org/10.1109/90. 532871

[12] Chaurasia S N and Singh A 2015 A hybrid swarm intelligence approach to the registration area planning problem. Inf. Sci. 302: 50-69

[13] Järv O, Ahas R and Witlox F 2014 Understanding monthly variability in human activity spaces: a twelve-month study using mobile phone call detail records. Transp. Res. Part C: Emerg. Technol. 38: 122-135, https://doi.org/10.1016/j.trc. 2013.11.003

[14] Wang P and Akyildiz F 2011 Spatial Correlation and mobility-aware traffic modeling for wireless sensor networks. IEEE/ACM Trans. Netw. 19(6): 1860-1873

[15] Wang D, Pedreschi D, Song C, Giannotti F and Barabási A L 2011 Human mobility, social ties, and link prediction. In: Proceedings of the 17th ACM SIGKDD International Conference on Knowledge Discovery and Data Mining, ACM., pp. 1100-1108, https://doi.org/10.1145/2020408. 2020581

[16] Coscia M, Rinzivillo S, Giannotti F and Pedreschi D 2012 Optimal spatial resolution for the analysis of human mobility. In: Proceedings of the 2012 IEEE/ACM International Conference on Advances in Social Networks Analysis and Mining, vol. 2, pp. 248-252, https://doi.org/10.1109/asonam. 2012.50

[17] Wesolowski A, Eagle N, Tatem A J, Smith D L, Noor A M, Snow R W and Buckee C O 2012 Quantifying the impact of human mobility on malaria. Science 338: 267-270, https:// doi.org/10.1126/science. 1223467

[18] Safa H, Pierre S and Conan J 2002 A built-in memory model for reducing location update cost in mobile wireless networks. Comput. Commun. 25: 1343-1353, https://doi.org/10. 1016/s0140-3664(02)00012-9

[19] Singh A P and Karnan M 2010 A dynamic location management scheme for wirless networks using cascaded correlation neural network. Int. J. Comput. Theory Eng. 2(4): 581-585, https://doi.org/10.7763/ijcte.2010.v2.205

[20] Zahran A H and Liang B 2007 A generic framework for mobility modeling and performance analysis in next-generation heterogeneous wireless networks. IEEE Commun. Mag. 45: 92-99, Retrieved from http://www.scopus.com/inward/ record.url?eid=2-s2.0-35348818786\&partnerID=40\&md5= b7fc861e319d6df92c63b289b2aa17f3

[21] Song C, Qu Z, Blumm N and Barabási A L 2010 Limits of predictability in human mobility. Science 327: 1018-1021, https://doi.org/10.1126/science. 1177170

[22] Vazquez-Prokopec G M, Bisanzio D, Stoddard S T, Paz-Soldan V, Morrison A C, Elder J P, et al 2013 Using GPS technology to 
quantify human mobility, dynamic contacts and infectious disease dynamics in a resource-poor urban environment. PLoS One 8, https://doi.org/10.1371/journal.pone.0058802

[23] Wang J, Zhang H, Toril M and Wille V 2007 Trial results of intelligent paging in GERAN. IEEE Commun. Lett. 11: 829-831, https://doi.org/10.1109/lcomm.2007.070510

[24] Zang H and Bolot J 2007 Mining call and mobility data to improve paging efficiency in cellular networks. In: Proceedings of the 13th Annual ACM International Conference on Mobile Computing and Networking - MobiCom '07, p. 123, https://doi.org/10.1145/1287853.1287868

[25] Misra A, Roy A and Das S K 2008 Information-theory based optimal location management schemes for integrated multisystem wireless networks. IEEE/ACM Trans. Netw. 16: 525-538, https://doi.org/10.1109/tnet.2007.901067

[26] Calabrese F, Diao M, Lorenzo G D, Ferreira J and Ratti C 2012 Understanding individual mobility patterns from urban sensing data: a mobile phone trace example. IRES Working Paper Series, https://doi.org/10.1016/j.trc.2012.09. 009
[27] Maitra M, Saha D, Bhattacharjee P S and Mukherjee A 2008 An intelligent paging strategy using rule-based AI technique for locating mobile terminals in cellular wireless networks. IEEE Trans. Veh. Technol. 57: 1834-1845, https://doi.org/ 10.1109/tvt.2007.907074

[28] Parija S, Nath N P, Sahu P K and Singh S 2015 Dynamic profile based paging in mobile communication. In: Proceedings of the International Conference on Microwave, Optical and Communication Engineering (ICMOCE), pp. 342-345

[29] Noulas A, Scellato S, Lathia N and Mascolo C 2012 Mining user mobility features for next place prediction in locationbased services. In: Proceedings of the IEEE International Conference on Data Mining, ICDM, pp. 1038-1043, https:// doi.org/10.1109/icdm.2012.113

[30] Do T M T and Gatica-Perez D 2012 Contextual conditional models for smartphone-based human mobility prediction. In: Proceedings of the 2012 ACM Conference on Ubiquitous Computing (UbiComp), p. 163, https://doi.org/10.1145/ 2370216.2370242 